\title{
Multiple Particle Tracking Detects Changes in Brain Extracellular Matrix and Predicts Neurodevelopmental Age
}

Michael McKenna ${ }^{1}$, David Shackelford ${ }^{1}$, Hugo Ferreira Pontes ${ }^{1}$, Brendan Ball ${ }^{1}$, Elizabeth Nance ${ }^{1-4,5 *}$

${ }^{1}$ Department of Chemical Engineering, University of Washington, Seattle WA 98195, United States

2 Department of Radiology, University of Washington, Seattle WA 98195, United States

${ }^{3}$ Center on Human Development and Disability, University of Washington, Seattle WA 98195, United States

${ }^{4}$ eScience Institute, University of Washington, Seattle WA 98195, United States

5 Lead Contact

Department of Chemical Engineering

University of Washington

105 Benson Hall

Box 351750

Seattle, WA 98195-1750, United States

*Corresponding author: eanance@uw.edu 


\section{Supporting Information}

\section{Supplemental Experimental Procedures}

\section{Organotypic hemispheric brain slice preparation}

Animals were administered an intraperitoneal injection of pentobarbital (150 mg/kg). After euthanasia, brains were rapidly removed and immersed in room temperature $\left(22^{\circ} \mathrm{C}\right)$ dissection media consisting of $500 \mathrm{~mL}$ HBSS (no $\mathrm{Mg}^{2+}$, no $\mathrm{Ca}^{2+}$, ThermoFisher, Waltham, MA), $1 \%$ Penicillin-Streptomycin (MilliporeSigma), and $3.2 \mathrm{~g}$ glucose (MilliporeSigma). Whole brains were cut into hemispheres with a razor blade, and $300 \mu \mathrm{m}$-thick coronal slices were prepared from each hemisphere using a Mcllwain tissue chopper. Slices were transferred to a Petri dish filled with room temperature dissection media and separated under a surgical dissection microscope using fine tip paint brushes. Individual slices containing corpus callosum but lacking hippocampus were isolated and placed on $30 \mathrm{~mm}$ cell culture inserts (Fisher Scientific) in nontreated 6-well plates (USA Scientific). Prior to plating, 6-well plates were filled with $1 \mathrm{~mL}$ slice culture media (SCM) containing $250 \mathrm{~mL}$ MEM (ThermoFisher, no glutamine, no phenol red), $125 \mathrm{~mL}$ HBSS (with $\mathrm{Mg}^{2+}$, with $\mathrm{Ca}^{2+}$, ThermoFisher), $125 \mathrm{~mL}$ horse serum (ThermoFisher), $5 \mathrm{~mL}$ GlutaMAX Supplement (Fisher Scientific), and 1\% Penicillin-Streptomycin. Slices were incubated in sterile conditions at $37^{\circ} \mathrm{C}$ and $5 \% \mathrm{CO}_{2}$.

\section{Nanoparticle preparation and characterization}

The covalent attachment of MeO-PEG- $\mathrm{NH}_{2}$ (5kDa MW, Creative PEG Works) to the surface of 40nm fluorescent PS-COOH nanoparticle (Fisher Scientific) was carried out using a carboxyl amine reaction. Briefly, $50 \mu \mathrm{L}$ of stock PS-COOH particle suspension was washed and resuspended to six-fold dilution in ultrapure water. A four-fold molar excess of MeO-PEG-NH was added to the particle suspension and mixed to dissolve the PEG. $N$ Hydroxysulfosuccinimide (NHS, MilliporeSigma) was added to a final concentration of $60 \mathrm{mM}$ and $200 \mathrm{mM}$ borate buffer ( $\mathrm{pH}$ 8.2) was added to dilute the $300 \mu \mathrm{L}$ sample volume five-fold. 1Ethyl-3-(3-dimethylaminopropyl) carbodiimide (EDC, Invitrogen, Carlsbad, CA) was added to stoichiometrically complement the MeO-PEG-NH${ }_{2}$. Tubes containing particle suspensions were wrapped in aluminum foil and placed on a rotary incubator for $6 \mathrm{~h}$ at room temperature $\left(22^{\circ} \mathrm{C}\right)$ and then washed twice via centrifugation (Amicon Ultra $0.5 \mathrm{~mL} 100 \mathrm{k}$ MWCO; MilliporeSigma) at $14,000 \times \mathrm{g}$ for $12 \mathrm{~min}$ at $4^{\circ} \mathrm{C}$. Particles were resuspended in ultrapure water to the initial stock particle volume and stored at $4^{\circ} \mathrm{C}$ until use. 
For nanoparticle characterization, both DLS and laser Doppler anemometry were performed using the Zetasizer Nano ZS (Malvern Instruments, Malvern, UK). Particles were diluted to $\sim 0.002 \%$ solids in filtered (0.45 um, Whatman, Maidstone, UK) $10 \mathrm{mM} \mathrm{NaCl}, \mathrm{pH} 7.0$, prior to measurement.

\section{Characterizing the timeline of enzyme-induced perineuronal net breakdown in organotypic rat brain slices ex vivo}

Slices were treated with either ChABC (MilliporeSigma), HYase (Fisher Scientific), or SCM (non-treated, NT) working solution. ChABC working solution was prepared by reconstituting Chondroitinase $\mathrm{ABC}$ to $0.4 \mathrm{U} / \mathrm{mL}$ in an aqueous buffer containing $50 \mathrm{mM}$ Tris $\mathrm{HCl}, \mathrm{pH} 8.0$ (ThermoFisher Scientific), and 50mM sodium acetate (MilliporeSigma). For the HYase working solution, HYase from Streptomyces hyalurolyticus (Fisher Scientific) was reconstituted to 35 $\mathrm{U} / \mathrm{mL}$ in $4^{\circ} \mathrm{C} 1 x$ Dulbecco's Phosphate-Buffered Saline (1x PBS, no $\mathrm{Mg}^{2+}$, no $\mathrm{Ca}^{2+}$, Corning). Following reconstitution, both $\mathrm{ChABC}$ and $\mathrm{HYase}$ working solutions were aliquoted and stored at $-20^{\circ} \mathrm{C}$ prior to use. The NT working solution consisted of SCM.

All working solutions were brought to room temperature $\left(22^{\circ} \mathrm{C}\right)$ prior to use. At the initial timepoint, $200 \mu \mathrm{L}$ of a given treatment solution was applied to the top of each brain slice in a dropwise fashion and returned to the incubator, where slices were maintained at $37^{\circ} \mathrm{C}$ and $5 \%$ $\mathrm{CO}_{2}$. At subsequent timepoints, a brain slice was removed and placed in $10 \%$ formalin phosphate buffer (Fisher Scientific) for fixation. One brain slice from each treatment group was removed at 15-, 30-, 60-, and 120-min post treatment, resulting in a total of 4 slices per treatment condition. Slices were incubated in formalin for $1 \mathrm{~h}$ at room temperature $\left(22^{\circ} \mathrm{C}\right)$, washed 2 times with $500 \mu \mathrm{L} 1 \times$ PBS for 5 minutes each, and stored at $4^{\circ} \mathrm{C}$ until staining commenced. Slices were stained within 1 week of fixation with $500 \mu \mathrm{L}$ of $1 \mathrm{x}$ PBS containing 10 $\mu \mathrm{g} / \mathrm{mL}$ WFA Lectin (Vector Laboratories Inc, Burlingame, CA) for $12 \mathrm{~h}$ at $4^{\circ} \mathrm{C}$. Following WFA incubation, slices were washed 2 times with $500 \mu \mathrm{L}$ 1x PBS for 5 minutes, and cell nuclei were stained with $500 \mu \mathrm{L}$ of $1 \times$ PBS containing $1 \mu \mathrm{g} / \mathrm{mL}$ DAPI (ThermoFisher) for $30 \mathrm{~min}$. Slices were subject to a final washing step and stored in $1 \times$ PBS at $4^{\circ} \mathrm{C}$ until imaged.

All imaging of fixed slices was performed using a confocal microscope (Nikon Instruments, Melville, NY). At a 20x magnification, 3 representative images were taken from the cortex of each brain slice. 


\section{Supplemental Figures and Tables}

Table S1. Physicochemical properties of the 40nm PS-PEG nanoparticles. Nanoparticle hydrodynamic diameter and PDI as determined by DLS. Laser Doppler anemometry was used to determine nanoparticle $\zeta$-potential. All experiments were performed at $25^{\circ} \mathrm{C}$ in $10 \mathrm{mM} \mathrm{NaCl}, \mathrm{pH}$ 7.0. Values represent the average \pm standard deviation of $n=3$ measurements.

\begin{tabular}{|l|l|l|l|}
\hline Sample & $\begin{array}{l}\text { Hydrodynamic } \\
\text { Size }(\mathbf{n m})\end{array}$ & PDI & $\zeta$-Potential $(\mathbf{m V})$ \\
\hline 40nm PS-PEG & $51 \pm 2$ & $0.027 \pm 0.018$ & $-0.59 \pm 0.10$ \\
\hline
\end{tabular}

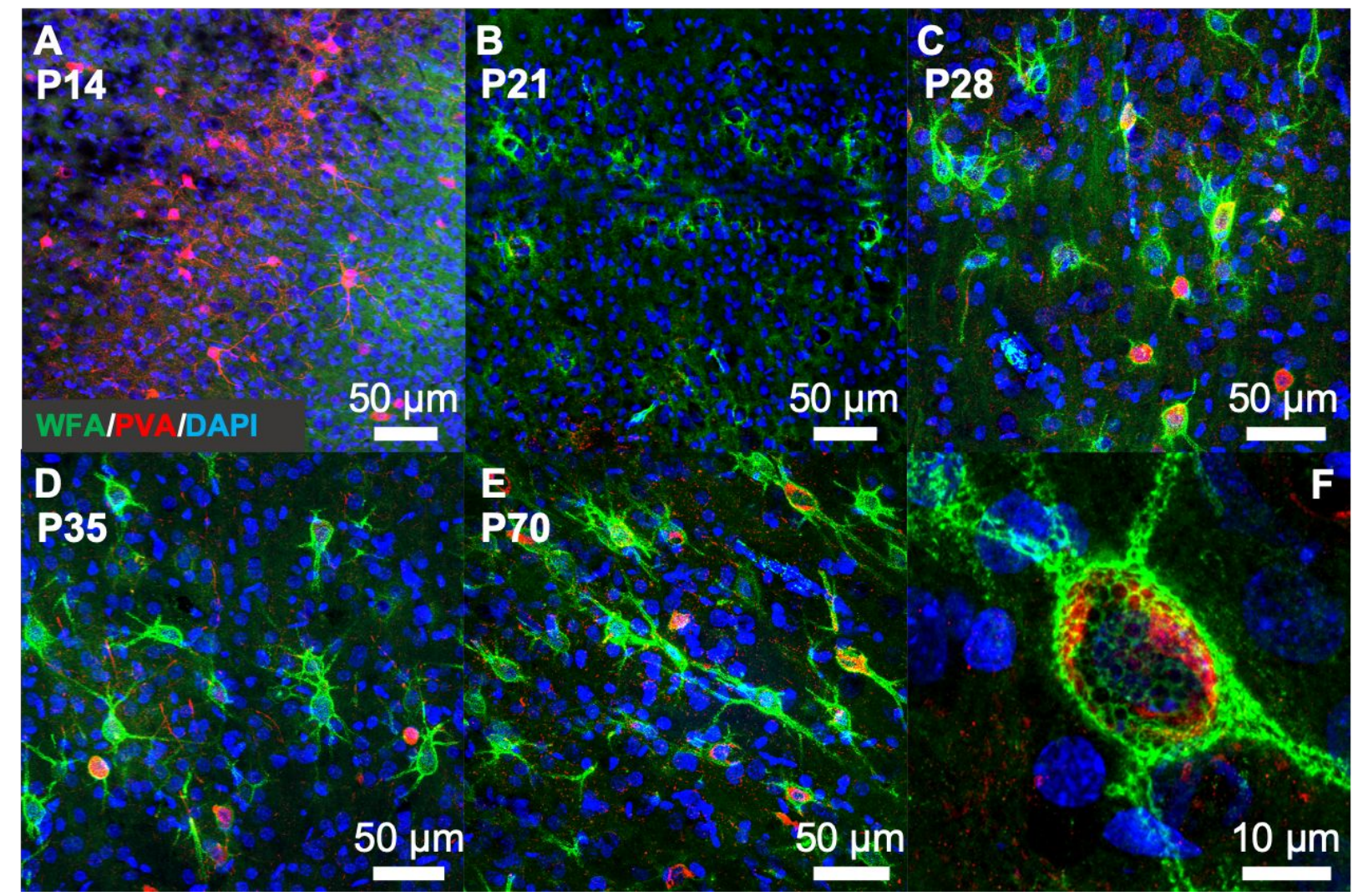

Figure S1. Individual slice MPT data following enzymatic treatment. (A-E) Representative images taken in the cortex of (A) P14, (B) P21, (C) P28, (D) P35, and (E) P70 rat brains. Formalin fixed brain sections were stained with WFA for PNNs (green) and anti-parvalbumin for a subpopulation of GABAergic interneurons (red). Cell nuclei were stained with DAPI (blue). Scale bar $=50 \mu \mathrm{m}$. (F) High-resolution image of PNN (green) enveloping a GABAergic interneuron (red). Scale bar $=10 \mu \mathrm{m}$. 
A

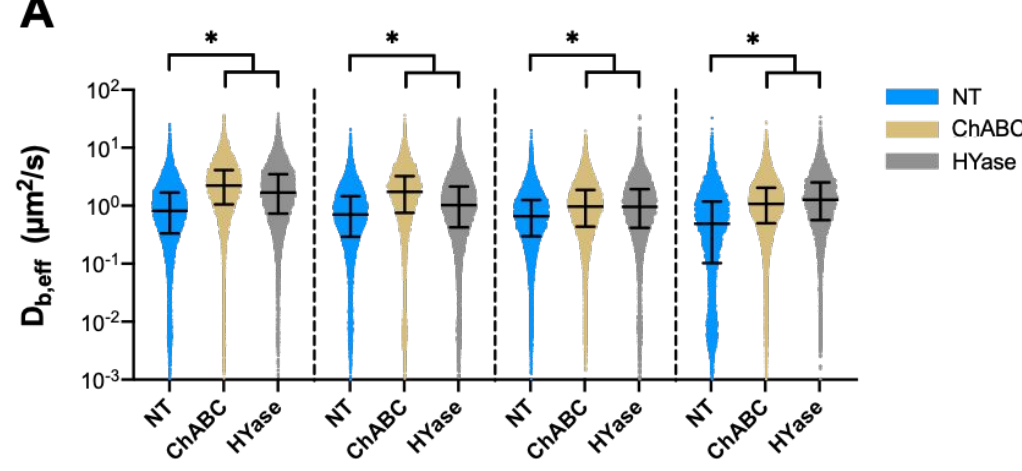

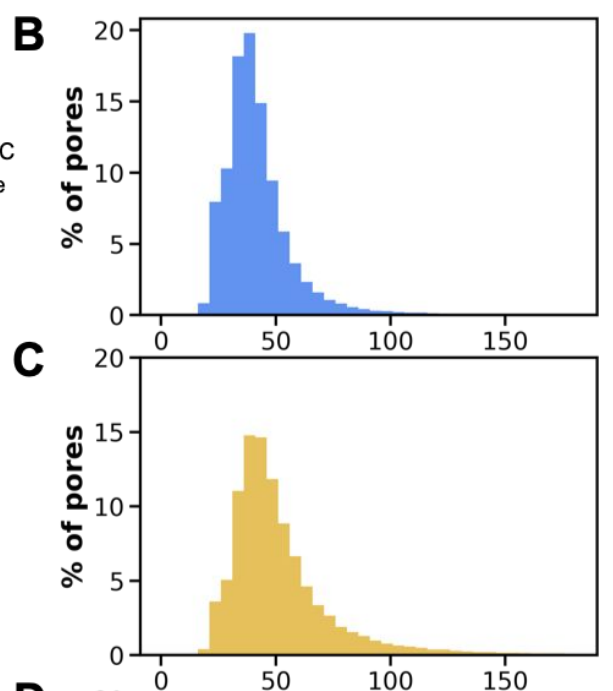

D

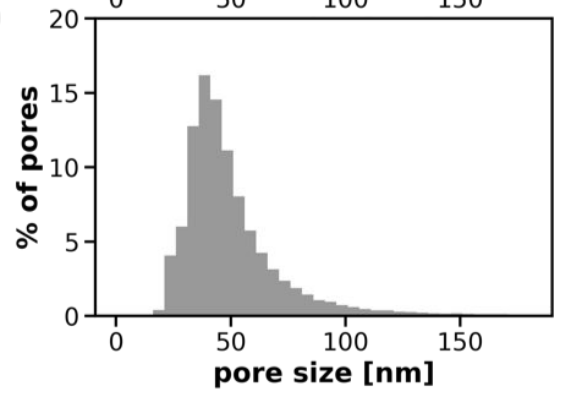

Figure S2. Individual slice MPT data following enzymatic treatment. (A) $D_{b, \text { eff }}$ distributions and (B-D) effective pore size distributions for enzyme-treated and non-treated P35 brain slices. Color scheme: NT=blue, ChABC-treated=gold, HYase-treated=grey. 
Table S2. A complete list of all 39 trajectory features calculated by the diff-classifier Python package. Included for each feature is a brief description and how it is determined. Additional documentation can be found in the TraJ GitHub repository (https://github.com/thorstenwagner/TraJ.git).

\begin{tabular}{|c|c|c|c|}
\hline Feature & $\begin{array}{l}\text { Model } \\
\text { Abbreviation }\end{array}$ & Description & How it is determined \\
\hline alpha $(\alpha)$ & alpha & Exponent of the anomalous diffusion equation. & $\begin{array}{l}\text { Non-linear least squares is used to fit raw } \\
\text { MSD vs. lag time }(\tau) \text { data to the } \\
\text { anomalous diffusion equation: } \\
\qquad M S D=4 D_{f i t} \tau^{\alpha}\end{array}$ \\
\hline $\begin{array}{l}\text { Effective } \\
\text { diffusion } \\
\text { coefficient } \\
\left(D_{\text {fit }}\right)\end{array}$ & $D_{-}$fit & $\begin{array}{l}\text { Coefficient of the anomalous diffusion } \\
\text { equation. }\end{array}$ & $\begin{array}{l}\text { Non-linear least squares is used to fit raw } \\
\text { MSD vs. lag time }(\tau) \text { data to the } \\
\text { anomalous diffusion equation: } \\
\qquad M S D=4 D_{f i t} \tau^{a}\end{array}$ \\
\hline Kurtosis $(K)$ & kurtosis & $\begin{array}{l}\text { The fourth moment of the projected positions } \\
\text { on the dominant eigenvector of the radius of } \\
\text { gyration tensor (T). }\end{array}$ & $K=\frac{1}{N} \sum_{i=0}^{N-1} \frac{\left(x_{i}^{p}-\overline{x^{p}}\right)^{4}}{\sigma_{x^{p}}^{4}}$ \\
\hline $\begin{array}{l}\text { Asymmetry } 1 \\
\text { (a) }\end{array}$ & asymmetry 1 & $\begin{array}{l}\text { Characterizes the asymmetry of the trajectory. } \\
\text { Asymmetry } 1 \text { equals } 0 \text { for circularly symmetric } \\
\text { trajectories and } 1 \text { for linear trajectories. }\end{array}$ & 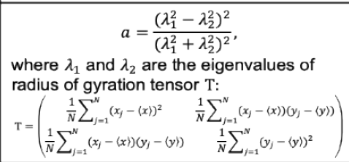 \\
\hline $\begin{array}{l}\text { Asymmetry2 } \\
\left(a_{2}\right)\end{array}$ & asymmetry2 & $\begin{array}{l}\text { The ratio of the smaller to larger principal } \\
\text { radius of gyration. }\end{array}$ & $a_{2}=\frac{\lambda_{2}}{\lambda_{1}}$ \\
\hline $\begin{array}{l}\text { Asymmetry3 } \\
\left(a_{3}\right)\end{array}$ & asymmetry3 & $\begin{array}{l}\text { An asymmetry feature that accounts for non- } \\
\text { cylindrically symmetric point distributions. }\end{array}$ & $a_{3}=-\log \left(1-\frac{\left(\lambda_{1}-\lambda_{2}\right)^{2}}{2\left(\lambda_{1}+\lambda_{2}\right)^{2}}\right)$ \\
\hline $\begin{array}{l}\text { Aspect ratio } \\
(A R)\end{array}$ & AR & $\begin{array}{l}\text { The ratio of the long and short side of the } \\
\text { trajectory's minimum bounding rectangle. } \\
\text { Perfectly symmetric trajectories have an } \\
\text { aspect ratio of } 1 \text {, and aspect ratio increases as } \\
\text { trajectories become more elongated }\end{array}$ & $A R= \begin{cases}\mid \frac{\left|x_{\max }-x_{\min }\right|}{\mid y_{\max }-y_{\min }}, & \left|y_{\max }-y_{\min }\right|<\left|x_{\max }-x_{\min }\right| \\
\frac{y_{\max }-y_{\min } \mid}{\mid x_{\max }-x_{\min },}, & \left|y_{\max }-y_{\min }\right| \geq\left|x_{\max }-x_{\min }\right|\end{cases}$ \\
\hline Elongation & elongation & $\begin{array}{l}\text { An estimation of amount of extension of the } \\
\text { trajectory from its centroid. }\end{array}$ & Elongation $=1-\left(\frac{1}{A R}\right)$ \\
\hline $\begin{array}{l}\text { Boundedness } \\
(B)\end{array}$ & boundedness & $\begin{array}{l}\text { Boundedness quantifies how much a particle } \\
\text { with diffusion coefficient } D_{\text {eff }} \text { is restricted by a } \\
\text { circular confinement of radius } r \text { when diffusing } \\
\text { for a period of time N } \Delta t \text {. }\end{array}$ & $B=\frac{D_{e f f} N \Delta t}{r^{2}}$ \\
\hline $\begin{array}{l}\text { Fractal } \\
\text { Dimension } \\
\left(D_{f}\right)\end{array}$ & fractal_dim & $\begin{array}{l}\text { Fractal dimension is a measure of how } \\
\text { "complicated" a self similar figure is. }\end{array}$ & $D_{f}=\frac{\log (N)}{\log \left(N d L^{-1}\right)}$ \\
\hline $\begin{array}{l}\text { Trappedness } \\
\left(p_{t}\right)\end{array}$ & trappedness & $\begin{array}{l}\text { The probability }\left(p_{t}\right) \text { that a particle with } \\
\text { diffusion coefficient } D_{\text {eff }} \text { is trapped in a region } \\
\left(r_{0}\right) \text { for a period of time } N \Delta t \text {. }\end{array}$ & $p_{t}=1-\exp \left(0.2048-0.25117\left(\frac{D_{\text {eff }} N \Delta t}{r_{0}^{2}}\right)\right)$ \\
\hline Efficiency $(E)$ & efficiency & $\begin{array}{l}\text { The ratio of squared net displacement to the } \\
\text { sum of squared step lengths. }\end{array}$ & $E=\frac{\left(x_{N-1}-x_{0}\right)^{2}+\left(y_{N-1}-y_{0}\right)^{2}}{\sum_{i=1}^{N-1}\left(x_{i}-x_{i-1}\right)^{2}+\left(y_{i}-y_{i-1}\right)^{2}}$ \\
\hline $\begin{array}{l}\text { Straightness } \\
(S)\end{array}$ & straightness & $\begin{array}{l}\text { The ratio of net displacement to the sum of } \\
\text { step lengths. }\end{array}$ & $S=\frac{\sqrt{\left(x_{N-1}-x_{0}\right)^{2}+\left(y_{N-1}-y_{0}\right)^{2}}}{\sum_{i=1}^{N} \sqrt{\left(x_{i}-x_{i-1}\right)^{2}+\left(y_{i}-y_{i-1}\right)^{2}}}$ \\
\hline $\begin{array}{l}\text { MSD Ratio } \\
\left(M S D_{n_{1}, n_{2}}\right)\end{array}$ & MSD_ratio & $\begin{array}{l}\text { MSD ratio characterizes the shape of the MSD } \\
\text { curve. For Brownian motion, it is } 0 \text {; For } \\
\text { restricted motion it is }<0 \text {; For directed motion } \\
\text { it is }>0 \text {. }\end{array}$ & $M S D_{n_{1}, n_{2}}=\frac{M S D_{n_{1}}}{M S D_{n_{2}}}-\frac{n_{1}}{n_{2}}$ \\
\hline Frames & frames & $\begin{array}{l}\text { The total number of frames the trajectory } \\
\text { spans. }\end{array}$ & Frames $=N$ \\
\hline $\begin{array}{l}\text { Mean Turning } \\
\text { Angle }\left(\Theta_{\text {mean }}\right)\end{array}$ & angle_mean & $\begin{array}{l}\text { The trajectory mean of the turning angle which } \\
\text { is the counterclockwise angle from one point } \\
\text { to another }\end{array}$ & $\frac{1}{N} \sum_{i=1}^{N} \theta_{i, i+1}$ \\
\hline $\begin{array}{l}\text { Mean Turning } \\
\text { Angle } \\
\text { Magnitude } \\
\left.\text { ( } \Theta_{\text {mag }}\right)\end{array}$ & $\begin{array}{l}\text { angle_mag_ } \\
\text { mean }\end{array}$ & $\begin{array}{l}\text { The trajectory mean of the magnitude turning } \\
\text { angle which is the counterclockwise angle } \\
\text { from one point to another }\end{array}$ & $\frac{1}{N} \sum_{i=1}^{N}\left|\theta_{i, i+1}\right|$ \\
\hline $\begin{array}{l}\text { Turning Angle } \\
\text { Variance }\left(\Theta_{\text {var }}\right)\end{array}$ & angle_var & $\begin{array}{l}\text { The trajectory variance of the turning angle } \\
\text { which is the counterclockwise angle from one } \\
\text { point to another }\end{array}$ & $\frac{\sum_{i=1}^{N}\left(\theta_{i, i+1}-\bar{\theta}\right)^{2}}{N}$ \\
\hline $\begin{array}{l}\text { Total Distance } \\
\left(\mathrm{d}_{\text {total }}\right)\end{array}$ & dist_tot & $\begin{array}{l}\text { Total distance particle travels throughout } \\
\text { trajectory }\end{array}$ & $d_{\text {total }}=\sum_{i=1}^{N} \sqrt{\left(x_{i}-x_{i-1}\right)^{2}+\left(y_{i}-y_{i-1}\right)^{2}}$ \\
\hline $\begin{array}{l}\text { Net Distance } \\
\left(d_{\text {net }}\right)\end{array}$ & dist_net & Net distance traveled throughout trajectory & $d_{n e t}=\sqrt{\left(x_{N}-x_{0}\right)^{2}+\left(y_{N}-y_{0}\right)^{2}}$ \\
\hline Progression & progression & $\begin{array}{l}\text { The ratio of the net distance traveled, and the } \\
\text { total distance traveled }\end{array}$ & progression $=\frac{d_{\text {net }}}{d_{\text {total }}}$ \\
\hline Deff1 & Deff1 & Effective diffusion coefficient at $0.33 \mathrm{~s}$. & Deff1 $=\frac{M S D_{\tau=0.1}}{4 * 0.1}$ \\
\hline Deff2 & Deff2 & Effective diffusion coefficient at $3.3 \mathrm{~s}$. & Deff2 $=\frac{M S D_{\tau=1}}{4 * 1}$ \\
\hline \multicolumn{4}{|c|}{$\begin{array}{l}\text { Mean values were calculated based on surrounding datapoints for alpha, D_fit, kurtosis, asymmetry1, asymmetry2, asymmetry3, } \\
\text { AR, elongation, boundedness, fractal_dim, trappedness, efficiency, straightness, MSD_ratio, Deff1, and Deff2 }\end{array}$} \\
\hline \multicolumn{3}{|c|}{$\begin{array}{l}\text { MSD: mean squared displacement } \\
N: \text { number of frames } \\
\sigma_{x^{p}}: \text { standard deviation of the projected } 2 \mathrm{D} \text { positions } \\
\lambda_{1}, \lambda_{2}: \text { eigenvalues of radius of gyration tensor } \\
D_{\text {eff }}: \text { :ffective diffusion coefficient } \\
r: \text { radius of circular confinement } \\
n: \text { frame number } \\
r_{0}: \text { radius of trapped region } \\
i_{\text {Back }}: \text { average background pixel intensity }\end{array}$} & $\begin{array}{l}\text { osition } \\
\text { and y location } \\
\text { ee rate } \\
\text { between any two positions } \\
\text { m over all steplengths) of trajectory } \\
\text { tion of pixel intensities }\end{array}$ \\
\hline
\end{tabular}


A

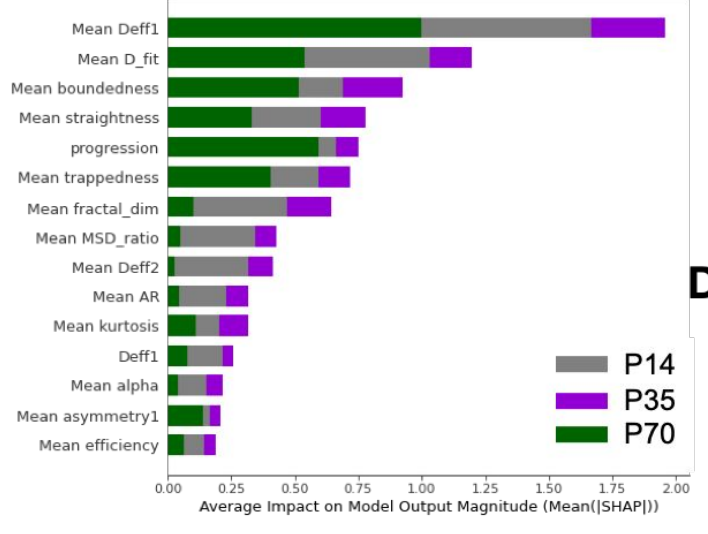

B

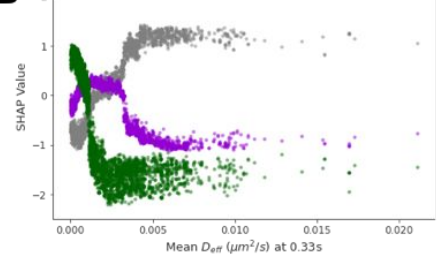

D

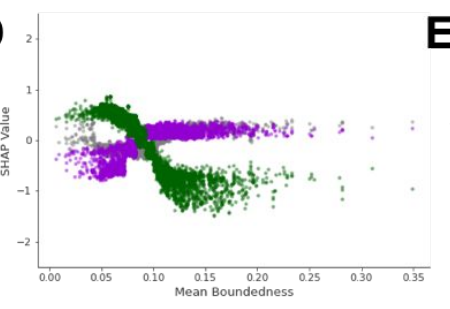

C

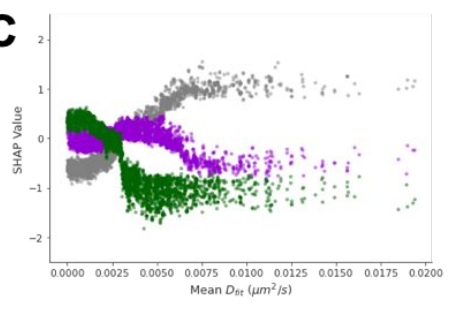

$\mathbf{E}$

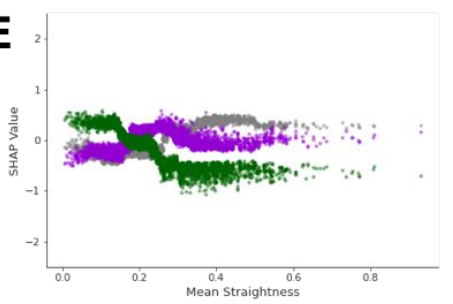

Figure S3. SHAP analysis using a reduced resolution model. (A) SHAP feature importance and (B-E) SHAP dependence plots for the four most influential features in the reduced resolution model. Displayed are SHAP dependence plots for (A) Mean $D_{\text {eff, }}(B)$ Mean $D_{\text {fit }}$, (C) Mean trappedness, and (D) Mean boundedness. Color scheme: P14=grey, P35=purple, P70=green.

A

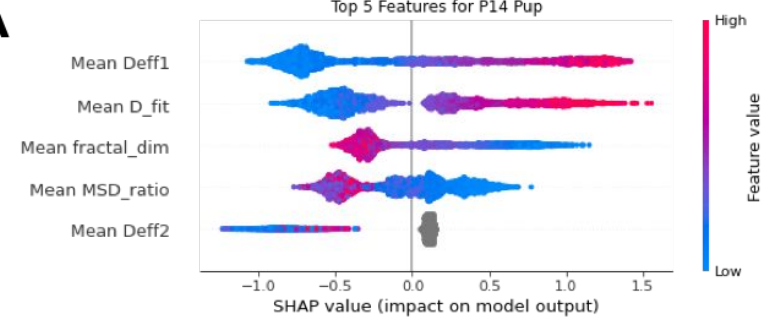

B

B Top 5 Features for P35 Pup
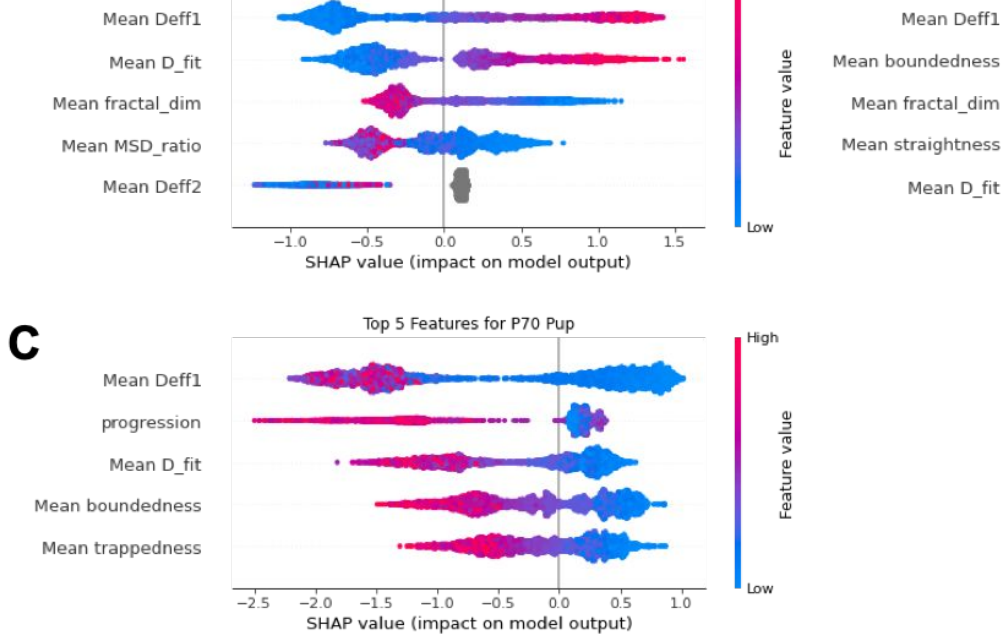

Figure S4. SHAP summary plots for each age of the 3-age boosted decision tree

classifier. SHAP summary plots of the five most influential features for predicting the (A) P14, (B) P35, and (C) P70 age groups. Colorbar provides feature magnitude from low (blue) to high (red). 